\title{
What will Gaia tell us about the Galactic disk?
}

\author{
Coryn A. L. Bailer-Jones \\ Max-Planck-Institut für Astronomie, Königstuhl 17, 69117 Heidelberg, Germany \\ email: calj@mpia.de
}

\begin{abstract}
Gaia will provide parallaxes and proper motions with accuracy ranging from 10 to 1000 microarcsecond on up to one billion stars. Most of these will be disk stars: for an unreddened $\mathrm{K}$ giant at $6 \mathrm{kpc}$, it will measure the distance accurate to $2 \%$ and the transverse velocity to an accuracy of about $1 \mathrm{~km} / \mathrm{s}$. Gaia will observe tracers of Galactic structure, kinematics, star formation and chemical evolution across the whole HR diagram, including Cepheids, RR Lyrae, white dwarfs, F dwarfs and HB stars. Onboard low resolution spectrophotometry will permit - in addition to an effective temperature estimate - dwarf/giant discrimination, metallicity measurement and extinction determination. For the first time, then, Gaia will provide us with a three-dimensional spatial/properties map and at least a two-dimensional velocity map of these tracers. (3D velocities will be obatined for the brighter sources from the onboard RV spectrograph). This will be a goldmine of information from which to learn about the origin and evolution of the Galactic disk. I briefly review the Gaia mission, and then show how the expected astrometric accuracies translate into distance and velocity accuracies and statistics. I then briefly examine the impact Gaia should have on a few scientific areas relevant to the Galactic disk, specifically disk structure and formation, the age-metallicity-velocity relation, the massluminosity relation, stellar clusters and spiral structure. Concerning spiral arms, I note how a better determination of their locations and pattern speed from their OB star population, plus a better reconstruction of the Sun's orbit over the past billion years (from integration through the Gaia-measured gravitational potential) will allow us to assess the possible role of spiral arm crossings in ice ages and mass extinctions on the Earth.
\end{abstract}

Keywords. space vehicles; telescopes; astrometry; stars: general; Galaxy: structure, stellar content, spiral arms; ice ages and mass extinctions

\section{Gaia overview}

Gaia is a high-accuracy astrometric satellite to be launched by ESA at the end of 2011. By measuring the positions of stars tens of times over a five year baseline, it can derive the mean position, the parallax and two-dimensional proper motion of each star. These are five components of the six dimensional phase space, the sixth component the radial velocity - being provided for the brighter stars by an onboard spectrograph. Gaia delivers absolute parallaxes tied to an inertial (quasar-based) reference frame. Gaia, which is currently under construction, represents a major step beyond the enormously successful Hipparcos mission, and is currently the only large-scale astrometric mission beyond the planning stage.

Gaia will perform a survey of the entire sky complete to magnitude $\mathrm{G}=20(\mathrm{~V}=20-22)$. This covers some $10^{9}$ stars, a million quasars and a few million galaxies. The sky coverage is complete to this magnitude, bar about $1 \%$ of the sky area where Gaia is confusion limited. Gaia will achieve an astrometric accuracy of $12-25 \mu$ as at $\mathrm{G}=15$ (providing a distance accuracy of $1-2 \%$ at $1 \mathrm{kpc}$ ) and $100-300 \mu$ as at $\mathrm{G}=20$. These numbers are also 
the approximate parallax accuracy in $\mu$ as and the proper motion accuracy in $\mu$ as/year. The accuracy range reflects a colour dependency: better accuracy is achieved for redder sources (as more photons are collected). Astrometry and photometry are done in a broad ("white light") band (G). Gaia will also measure radial velocities to a precision of $1-15 \mathrm{~km} / \mathrm{s}$ for stars with $\mathrm{V}=17$ via $\mathrm{R}=11500$ resolution spectroscopy around the CaII triplet (the "Radial Velocity Spectrograph", RVS). To characterize all sources (which are detected in real time), each is observed via low dispersion prism spectrophotometry over 330-1000 nm with a dispersion between 3 and $30 \mathrm{~nm} /$ pixel (the "BP/RP" instrument). From this we will estimate the "usual" astrophysical parameters, $\mathrm{T}_{\text {eff }}, \log g$ and $[\mathrm{Fe} / \mathrm{H}]$, but also the line-of-sight extinction to stars individually, plus perhaps also $[\alpha / \mathrm{Fe}]$ in some cases. Spectra from the radial velocity spectrograph will help the parameter determination for some stars brighter than about $V=14$, and will also allow the detection of emission lines and abundance anomalies.

Gaia has a nominal mission duration of five years (plus a possible one year extension), and 2-3 years following the end of operations are planned to complete the data processing. (The astrometry is self-calibrating, so the data must be reduced globally/simultaneously to get the final solutions and best astrometric accuracy.) The final catalogue will be available in about 2020, proceeded by earlier data releases. For more information on the satellite, science and data processing see http://www.rssd.esa.int/Gaia and the proceedings volume by Turon et al. (2005) (also available from the website).

\section{Distance and velocity accuracy and statistics}

Distances are vital in every area of astronomy. We need them to convert $2 \mathrm{D}$ angular positions to 3D spatial coordinates, allowing us to reveal the internal structure of stellar clusters or map the location of the spiral arms, for example. Knowing the distance we can convert 2D (angular) proper motions to physical velocities, and the apparent luminosity to the intrinsic luminosity, a fundamental quantity in stellar structure and evolution studies. Parallaxes are the only method of direct distance determination which does not make assumptions about the target source. We can measure parallaxes to virtually anything (not just, say, eclipsing binaries) and virtually all other rungs in the distance ladder are ultimately calibrated by them.

\subsection{How accuracy varies with distance}

Apart from very bright sources, the parallax error from Gaia is dominated by photon statistics. Thus the parallax uncertainty, $\sigma(\varpi)$, varies with the measured flux, $f$, as $\sigma(\varpi) \propto 1 / \sqrt{f}$. Therefore, for a source of given intrinsic luminosity, the parallax error is linearly proportional to distance, $\sigma(\varpi) \propto d$. The parallax itself of course also decreases with increasing distance, $\varpi=1 / d$, so the fractional parallax error, $\sigma(\varpi) / \varpi$, increases as distance squared, $\sigma(\varpi) / \varpi \propto d^{2}$. Converting a parallax to a distance is actually nontrivial, due to the need to account for Lutz-Kelker biases (see Binney \& Merrifield 1998 for a discussion). However, we can roughly equate this fractional parallax error with the fractional distance error, hereafter abbreviated as fde.

The proper motion uncertainty varies with distance in the same way as the parallax, and the measured proper motion itself, for given space velocity, decreases linearly with increasing distance. Therefore, the transverse velocity uncertainty (not fractional), also increases with the square of the distance, $\sigma(v) \propto d^{2}$ (for a source with fixed $M_{V}$ ). A useful relation to remember is that a proper motion of 1 mas/yr for a source at $1 \mathrm{kpc}$ corresponds to a transverse velocity of about $5 \mathrm{~km} / \mathrm{s}$. (Note that this is by definition 
because the parallax definition is related to the mean velocity of the Earth around the Sun.)

\subsection{Accuracies for certain types of stars}

The above relations are useful, because they enable us to see to what distance certain Galactic structure tracers can still be observed with useful accuracy. Here are some examples. A K1 giant, with an intrinsic luminosity of $M_{V}=1.0$, will have an apparent magnitude of about $G=15$ when at a distance of $6 \mathrm{kpc}$ (assuming zero interstellar extinction). (For simplicity I ignore the colour dependence of both the $V$ to $G$ relationship and the astrometric accuracy, adopting the lower accuracy blue limit for the latter.) The fde is $1.5 \%$ and $\sigma(v)=0.75 \mathrm{~km} / \mathrm{s}$. This clearly allows us to trace this tracer with high accuracy in phase space.

The astrometric accuracy, e.g. $\sigma(\varpi)$, is a factor of $\sqrt{10}$ worse for a source which is $2.5 \mathrm{mag}$ fainter; similarly the astrometric accuracy is a factor of two worse for a source which is $1.5 \mathrm{mag}$ fainter (equivalent to doubling the distance for a source of constant $\left.M_{V}\right)$.

A G3 dwarf with $M_{V}=5.0$ has $G=16.5$ at a distance of $2 \mathrm{kpc}$, and so a parallax accuracy of about $40 \mu \mathrm{as}$. The parallax itself is $500 \mu \mathrm{as}$, so the fde is $8 \%$. The transverse velocity accuracy is just $0.4 \mathrm{~km} / \mathrm{s}$ Moving it to $4 \mathrm{kpc}$ reduces the accuracy and parallax to $80 \mu$ as and $250 \mu$ as respectively, giving an fde of $30 \%$.

The velocity accuracies in particular are very good when one considers typical velocities within the Galaxy (e.g. the rotation of the LSR about the Galactic centre is about $220 \mathrm{~km} / \mathrm{s}$ ). So as the distance increases, it is parallaxes rather than the proper motions which first become less useful, although at large distances the transverse velocity uncertainty becomes dominated by the parallax error. The distance at which the parallax error is $50 \%$ is $5 \mathrm{kpc}$ for the G3 dwarf and $35 \mathrm{kpc}$ for the $\mathrm{K} 1$ giant, where the latter has an apparent magnitude of $G=18.8$. Thus we see that beyond a distance of about $40 \mathrm{kpc}$ (depending on intrinsic magnitude), the Gaia parallaxes on individual objects are no longer much good, so we will have to rely on spectroscopic parallaxes (derived from the onboard spectrophotometry), in particular to convert proper motions to velocities.

\subsection{Accuracy statistics}

While it's easy to throw out these numbers, we must not forget the enormous improvement they represent in accuracy above what is currently possible. The improvement with respect to Hipparcos comes primarily through the larger field of view: Gaia simulaneously observes tens of thousands of stars on a large CCD focal plane, whereas Hipparcos could only observe one at a time (with a photomultiplier tube, PMT) (Lindegren 2005). In addition, the larger QE detector (ca. 75\% for CCDs compared to around $1 \%$ for a PMT), the larger collecting area $\left(0.7 \mathrm{~m}^{2}\right.$ compared to $\left.0.03 \mathrm{~m}^{2}\right)$ and the broader effective bandpass together provide a much larger detectable photoelectron flux per star.

The value of Gaia is not just accuracies of individual objects, but also the fact that it is an all sky survey with a relatively deep limiting magnitude $(G=20)$, so these accuracies are achieved on large numbers of objects. (Note that the bright - saturation - limit of Gaia is $G=6$, so it can just reach stars visible to the naked eye.) Roughly how many can be found via a Galaxy model. We find that Gaia will provide distances with an accuracy of $1 \%$ or better for 11 million stars. Taking $12 \mu$ as as the lower limit to the Gaia parallax accuracy, this means that all of these stars must lie within 800 pc (i.e. have a parallax less than $1250 \mu \mathrm{as})$. Some 150 million stars will have a distance accuracy better than $10 \%$ (all within $8 \mathrm{kpc}$ ) and some 100000 stars better than $0.1 \%$ (all within $80 \mathrm{pc}$ ). For 
comparison, currently fewer than 200 stars have had their parallaxes measured to better than $1 \%$. All are within $10 \mathrm{pc}$ and all were measured with Hipparcos.

\section{Astrophysical parameter estimation}

Gaia does not use an input catalogue, so the properties of the sources are not known a priori. Gaia only observes point sources and the onboard processing generally bins the CCD data around a source in one-dimension (so that only the higher spatial resolution along-scan direction remains). Consequently there is little morphological information (although some can be reconstructed from the multiple scans of a given source which occur at a range of positions angles). There are, therefore, two main classification tasks with the Gaia data. The first is a discrete source classification, to discriminate between stars, galaxies and quasars (and perhaps also unresolved binaries and other types). Identification of a clean quasar sample (Bailer-Jones \& Smith 2008) is necessary in particular for the global astrometric solution.

The second step is to estimate the stellar astrophysical parameters (APs). This is done primarily using the low resolution prism spectrophotometry $(\mathrm{BP} / \mathrm{RP})$, as well as the parallax when available. The classification software is still under development, but we have preliminary estimates of the performance of a stellar parametrizer based on support vector machine classifiers applied to synthetic spectra convolved with a Gaia instrument and noise model. At $G=15$, the RMS uncertanties in the derived APs are as follows: $1-5 \%$ in $\mathrm{T}_{\text {eff }}$ for a wide range of effective temperatures (OB to $\mathrm{K}$ stars); line-of-sight extinction, $A_{V}$, to $0.05-0.1$ mag for hot stars; $<0.2$ dex in $[\mathrm{Fe} / \mathrm{H}]$ for spectral types later than $\mathrm{F}$ down to -2.0 dex metallicity; $\log g$ to between 0.1 and 0.4 dex for all luminosity classes, but to better than 0.1 dex for hot star (OBA stars). It should be noted that the performance varies a lot with the physical parameters themselves, as well as with $G$ magnitude (i.e. signal-to-noise ratio). Work in this area is ongoing.

The inferred stellar parameters are tied, of course, to the stellar models used to simulate the synthetic spectra on which the classification models are trained. (If the models are instead trained directly on real spectra, then these must somehow be parametrized using models, so our results are always tied to physical stellar models). In addition to developing classification algorithms, the Gaia DPAC (Data Processing and Analysis Consortium) is also working on improving the stellar input physics and model atmospheres. A groundbased observing programme will provide some data to calibrate the fluxes of the models.

\section{Gaia science}

I now outline a few areas of disk astrophysics where Gaia should make significant contributions.

\subsection{Disk structure}

Many aspects of the thin and thick disk structure are not well known (see Vallenari et al. 2005 for a summary). For example, thin disk scale height estimates vary between 200 and $330 \mathrm{pc}$. The inferred scale length varies between $2-3 \mathrm{kpc}$ (as measured by star counts and integrated light) and $2-4 \mathrm{kpc}$ (as measured by kinematics). The vertex deviation and the vertial tilt are not well known. All of these measurements of the velocity elipsoids are based on relatively small samples. Gaia will provide much larger samples with 3D spatial structure and $2 \mathrm{D}$ or $3 \mathrm{D}$ velocity measures. Transverse velocities accurate to about $1 \mathrm{~km} / \mathrm{s}$ will be achieved for some 50 million stars out to a few $\mathrm{kpc}$. 


\subsection{Substructure and mergers}

One of the most important questions Gaia will address is that of how and when the Galaxy formed. $\Lambda$ CDM models of galaxy formation predict that galaxies are built up by the hierarchical merger of smaller components and some suggest that the halo is composed primarily of merger remnants (for a review see Freeman \& Bland-Hawthorn 2002). ¿From extragalactic observations there is good evidence for both the accretion of small components and for the merging of similar-sized galaxies. Within our own Galaxy, recent surveys - 2MASS and SDSS in particular - have found the fossils of past and ongoing mergers in the halo and possibly also the disk of our Galaxy. They have been identified primarily as spatial overdensities in two-dimensional (angular) photometric maps of large areas of the sky (sometimes accompanied with radial velocities). In a few cases, distance measures have been included by taking magnitude as a distance proxy (Belokurov et al. 2006) or by examining the 2D density of a limited range of spectral types (i.e. using a spectroscopic parallax of some tracers) (Yanny et al. 2000). But because merging satellites are disrupted by the Galactic potential and the material spread out after several orbits, density maps are a limited means of finding substructure. Without an accurate distance the interpretation of $2 \mathrm{D}$ maps is plagued by projection effects. Even with perfect $3 \mathrm{D}$ maps, the contrast against the background (including other streams) is often low (Brown et al. 2005). To improve this, we need 3D kinematics, i.e. radial velocities and proper motions (combined with distances). In an axisymmetric potential the component of angular momentum parallel to this axis $\left(L_{z}\right)$ of a merging satellite is an integral of motion. In a static potential, the energy $(E)$ is also an integral of motion (Binney \& Tremaine 1987). Thus while a merging satellite could be well-mixed spatially, it would remain (approximately) unperturbed in $\left(L_{z}, E\right)$ space. Of course, the Galactic potential is neither time-independent nor perfectly axisymmetric and Gaia has measurement errors, but simulations have demonstrated that Gaia will be able to detect numerous streams $10 \mathrm{Gyr}$ or more (i.e. many orbits) after the start of their disruption (Helmi \& de Zeeuw 2000).

Gaia will perform a 5D phase space survey over the whole sky, with the sixth component - radial velocity - being available for stars brighter than $\mathrm{V}=17$. At this magnitude, spectral types A5III, AOV and K1III (which all have $\mathrm{M}_{V} \simeq 1.0$ ) are seen at a distance of $16 \mathrm{kpc}$ (for zero extinction). The corresponding proper motion accuracy is about $50 \mu \mathrm{as} / \mathrm{yr}$, or $4 \mathrm{~km} / \mathrm{s}$. In addition to the $5 \mathrm{D}$ or $6 \mathrm{D}$ phase space information, Gaia provides abundances and ages for individual stars. Search for patterns in this even higher dimensional space permits an even more sensitive (or reliable) search for substructure.

\subsection{Dark matter}

Two distinct aspects of the Gaia mission permit us to study the mass and distribution of dark matter in our Galaxy. First, from the 3D kinematics of selected tracer stars, Gaia will map the total gravitational potential (dark and bright) of our Galaxy, in particular the disk. Second, from its parallaxes and photometry, Gaia will make a detailed and accurate measurement of the stellar luminosity function. This may be converted to a (present-day) stellar mass function via a Mass-Luminosity relation (see section 4.5). From this we can infer a stellar mass distribution. Subtracting this from the total mass distribution obtained from the kinematics yields the dark matter distribution.

\subsection{Stellar structure and stellar clusters}

Stellar luminosity is a fundamental property, so its measurement across a range of masses, ages and abundances is a critical ingredient for testing and improving stellar models. In open and globular clusters an accurate determination of luminosities and effective 
temperatures (which Gaia also provides) gives us the HR diagram for different stellar populations. (To derive an accurate luminosity we also need an accurate estimate of the line-of-sight extinction. This will be obtained star-by-star from the Gaia spectrophotometry.) We may then address fundamental questions of stellar structure, such as the bulk Helium abundance (which is not observable in the spectrum), convective overshooting and diffusion. One of the main uncertainties in the age estimation in clusters is accurately locating the main sequence (for open clusters) or main sequence turn off (for globular clusters). Gaia's accurate parallaxes and unbiased (magnitude-limited) survey will greatly improve this.

In addition to using clusters as samples for refining stellar structure and evolution, we can also study them as populations. Gaia will observe many hundreds of clusters, allowing us to determine the (initial) mass function into the brown dwarf regime and examine its dependence on parameters such as metallicity, stellar density and environment. There are perhaps 70 open clusters and star formation regions with 500pc. For these, Gaia will provide individual distances to stars brighter than $\mathrm{G}=15$ to better than $1 \%$. This will permit us, for the first time, to map the 3D spatial structure of many clusters, with a depth accuracy as good as $0.5-1$ pc for clusters at 200 pc. From the $3 D$ kinematics we can likewise study the internal dynamics of a cluster. A $G=15$ star will have its proper motion measured with an accuracy of $20 \mu \mathrm{as} / \mathrm{yr}$, corresponding to a speed uncertainty of $0.1 \mathrm{~km} / \mathrm{s}$ at this distance (half this for a red star). The speed uncertainty varies linearly with the distance for a fixed magnitude, so at $200 \mathrm{pc}$ the speed uncertainty is just $20 \mathrm{~m} / \mathrm{s}$. With this accuracy we can measure the internal kinematics of the cluster and so investigate the phenomena of mass segregation, low mass star evaporation and the dispersion of clusters into the Galactic field. (I have assumed that the uncertainty in the transverse velocity is dominated by the proper motion error and not the distance error. For the sake of the proper motion to velocity conversion we can assume all stars to be at the same distance, and therefore average over many stars to get a more accurate distance.)

At larger distances Gaia will be able to say less about the internal structure of an open cluster. However, using the proper motions to help define membership, the average distance to the cluster, plus its age and metallicity, can be measured accurately. In this way, we can use a few thousand open clusters over distances of tens of kpc as tracers of the disk abundance gradient.

Just as Gaia should be able to identify the fossils of past mergers from their phase space substructure (section 4.2), so the 6D phase space data plus astrophysical parameters for tens of millions of stars will allow Gaia to detect new stellar clusters, associations or moving groups based on their clustering in a suitable multi-dimensional parameter space. It can likewise confirm or refute the existence of controversial clusters.

\subsection{Stellar mass-luminosity relation}

Gaia will detect many binary systems. These are found in a number of ways, as summarized by Arenou \& Söderhjelm (2005). Most are found via the astrometry as astrometric solutions which do not fit the standard 5-parameter model. These include both Keplerian fits to the data (for periods up to 10 years) and nonlinear motions (accelerated proper motions, periods up to a few hundred years). Very wide binaries can be found as common proper motion pairs. In addition, bright binaries can be found with the radial velocity spectrograph and as eclipsing binaries. Unresolved binaries of different spectral types with brightness ratios not too far from unity can be found as part of the classification work, from the identification of two spectral energy distributions.

For those systems with orbital periods of about ten years or less, Gaia can solve for the orbital elements and for the total mass of the system. If the components of the system 
are spatially resolved then we may determine their individual masses. Gaia furthermore measures accurate intrinsic luminosities. Together these allow us to determine the stellar Mass-Luminosity relation, and to do it with more stars and over a wider mass range that has yet been performed. (As Johannes Andersen pointed out after my talk, it may not be useful to talk about achieving an accurate Mass-Luminosity relation because such a thing does not exist, in the sense that the luminosity of a star is not uniquely determined by its mass, but also by abundance, rotation etc. Gaia could nonetheless shed some light on these extra dependencies.)

\section{Spiral arms, ice ages and mass extinctions}

Several papers have reported on a correlation and possible causal connection between spiral arm passages and ice ages and/or mass extinctions on the Earth. One possible mechanism is the exposure to massive stars in star forming regions: the increased cosmic ray flux from type Ib/II supernovae within 10 pc during such passges could increase terrestrial cloud cover (through water drop nucleation) and thus lower global temperatures for millions of years (e.g. Ellis \& Schramm 1995), and/or the OB star UV flux could destroy the ozone layer and cause widespread extinction. A second mechanism is that the passage through regions of larger stellar and gas density found in spiral arms could perturb the Oort cloud and send minor bodies into the inner solar system, where they could impact on the Earth.

Through accurate measurements of the 3D kinematics of sars, Gaia will map the gravitational potential of the Galaxy and accurately determine the velocity of the Sun. Via numerical integration we can then reconstruct the path of the Sun through the Galaxy over the last few hundred million years and examine whether past ice ages and mass exinctions coincide with spiral arms passages. This has been done by Gies \& Helsel (2005) using the potential and solar motion derived from Hipparcos by Dehnen \& Binney $(1998 \mathrm{a}, \mathrm{b})$. While they found some correlation between arm passages and ice ages, the results depended heavily on the poorly known position and velocity (pattern speed) of the spiral arms.

With Gaia we can dramatically improve this analysis: the astrometric accuracies are better than Hipparcos by a factor of 500 (12-25 $\mu$ as compared to $1000 \mu$ as), it includes many more stars ( 1 billion compared to 120 000) and extends to fainter magnitudes (20 rather than 12.4). With these data we can determine the gravitational potential at higher spatial resolution and therefore reconstruct the solar motion more accurately. Moreover, we can measure the position and velocities of the spiral arms themselves from the Gaia observations of their OB star population without assuming a rotation curve or needing to know the extinction. For an $\mathrm{OB}$ star at a distance of $4 \mathrm{kpc}$ from the Sun observed through 4 magnitudes of extinction, Gaia will determine its distance to an accuracy of $13 \%$, its space velocity to about $1 \mathrm{~km} / \mathrm{s}$. Gaia can do this for some $50000 \mathrm{OB}$ stars within a few kpc, allowing to build a more accurate model for the spiral arm kinematics and thus make more conclusive statements about the correlation between arm passages and Earth cataclysms.

Finally, Gaia will make a survey of Near-Earth Objects (NEOs). Gaia does real-time onboard object detection, so is sensitive to transient phenomena and fast moving objects. Gaia is predicted to detect some 16000 NEOs (Mignard 2002). While ground-based surveys will discover many more in the coming years, Gaia can derive accurate orbits and is sensitive to parts of the orbital parmeter space which cannot easily be reached 
from the ground. It should make a significant contribution to the census of potential Earth impactors.

\section{References}

Arenou, F. \& Söderhjelm, S. 2005, in: Turon, C., O'Flaherty, K. S., Perryman, M. A. C. (eds.), The Three-Dimensional Universe with Gaia, ESA, SP-576, p. 557

Bailer-Jones, C. A. L., Smith, K. S., Gaia Technical Note, GAIA-C8-TN-MPIA-CBJ-036, available from http://www.mpia.de/GAIA

Belokurov, V., Zucker, D. B., \& Evans, N. W., et al. 2006, ApJ 642, L137

Binney, J. \& Merrifield, M. 1998, Galactic Astronomy, Princeton University Press

Binney, J. \& Tremaine, S. 1987, Galactic Dynamics, Princeton University Press

Brown, A. G. A, Velázquez, H. M. \& Aguilar, L. A. 2005, MNRAS 359, 1287

Dehnen, W. \& Binney J. 1998a, MNRAS 294, 429

Dehnen, W. \& Binney J. 1998b, MNRAS 298, 387

Ellis, J. \& Schramm, N. 1995, Proc. Natl. Acad. Sci. 92, 235

Freeman, K. \& Bland-Hawthorn, J. 2002, ARAA 40, 487

Gies, D. R. \& Helsel, J. W. 2005, ApJ 626, 844

Helmi, A. \& de Zeeuw, P. T. 2000, MNRAS 319, 657

Migarnd, F. 2002, A\&A 393, 727

Lindegren, L. 2005, in: Turon, C., O'Flaherty, K. S., Perryman, M. A. C. (eds.), The ThreeDimensional Universe with Gaia, ESA, SP-576, p. 29

Turon, C., O'Flaherty, K. S., \& Perryman, M. A. C. (eds.), 2005, The Three-Dimensional Universe with Gaia, ESA, SP-576

Vallenari, A., Nasi, E., Bertelli, G., \& Chiosi, C., S. 2005, in: Turon, C., O'Flaherty, K. S., Perryman, M. A. C. (eds.), The Three-Dimensional Universe with Gaia, ESA, SP-576, p. 113

Yanny, B., Newberg, H., \& Kent, S., et al. 2000, ApJ 540, 825 